\title{
Integrative Analysis of miRNA Expression Profiling of Diabetic Foot Ulcers Wound Treated With Zizhu Ointment
}

\section{Xiaoming $\mathrm{Hu}$}

Shuguang Hospital

\section{Renyan Huang}

Shuguang Hospital

\section{Wenhui Li}

Shanghai University of Medicine and Health Sciences

\section{Xiao Yang}

Shuguang Hospital

\section{Weijing Fan}

Shuguang hospital

\section{Fang Guo}

Shanghai Jiao Tong University School of Life Sciences and Biotechnology

\section{Guobin Liu( $\nabla$ xgwk666@126.com )}

Shanghai university of traditional Chinese medicine https://orcid.org/0000-0002-3283-4275

\section{Research}

Keywords: diabetic foot ulcers, miRNA-sequence, wound healing, Zizhu ointment

Posted Date: January 4th, 2021

DOl: https://doi.org/10.21203/rs.3.rs-136736/v1

License: (c) (i) This work is licensed under a Creative Commons Attribution 4.0 International License. Read Full License 


\section{Abstract}

Background: A major complication of diabetes is diabetic foot ulcers. Millions of patients suffer from the physiological and psychosocial effects of diabetic foot ulcers. However, the current treatment is not effective, and the patients' prognosis has not been significantly improved.

Results: Traditional Chinese medicine-Zizhu ointment exhibited its own characteristics and advantages in treating diabetic foot ulcer by promoting wound healing. The mechanism of Zizhu ointment promoting diabetic foot ulcer wound healing was explored by detecting its miRNA expression profiling. Eighty-three differentially expressed miRNAs were identified, 30 of which were upregulated and 53 were downregulated in diabetic cutaneous wounds treated with Zizhu ointment. The Gene Ontology (GO) and Kyoto Encyclopedia of Genes and Genomes (KEGG) enrichment analysis of the differentially expressed miRNAs showed that they were involved in several pathways that could regulate the proliferation, differentiation, apoptosis, migration, and secretion of tissue repair cells, and were linked to inflammatory cells, the extracellular matrix, growth factors, and other relevant factors related to wound healing. Furthermore, miRNA-Target regulatory network and protein-protein interaction network were constructed. Twenty hub genes of networks were obtained.

Conclusions: Our preliminary study provides a new alternative drug treatment for diabetic foot ulcers by traditional Chinese medicine, and will assist in better understanding its therapeutic targets.

\section{Full Text}

This preprint is available for download as a PDF.

\section{Figures}


A
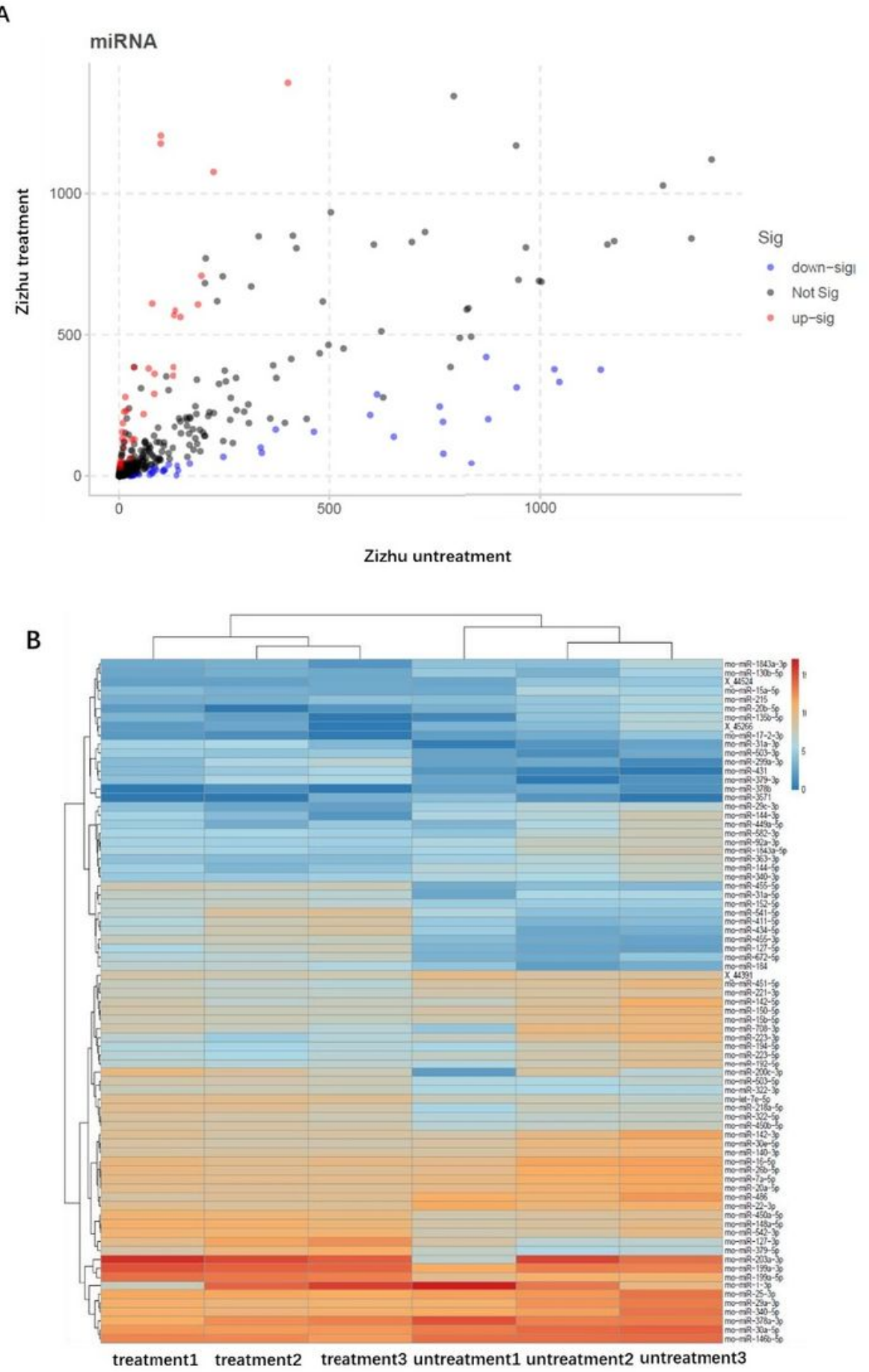

\section{Figure 1}

Visual analysis of differentially expressed miRNAs. (A) Volcano map of differentially expressed miRNAs . Each point in the graph represents a specific miRNA. The red points represent upregulated miRNAs with significance of $p<0.05$. The blue points represent downregulated miRNAs with significance of $p<0.05$. The black points are nonsignificantly differentially expressed miRNAs. (B) Differential miRNA expression clustering patterns. The columns in the diagram represent Zizhu ointment treated and untreated groups, 
and each row represents a miRNA. The colours represent relative expression of the miRNAs in the group, with red and blue representing upregulation and downregulation, respectively. The trend of expression is shown in the colour bar at the top right. The left side is a dendrogram of miRNA clustering.
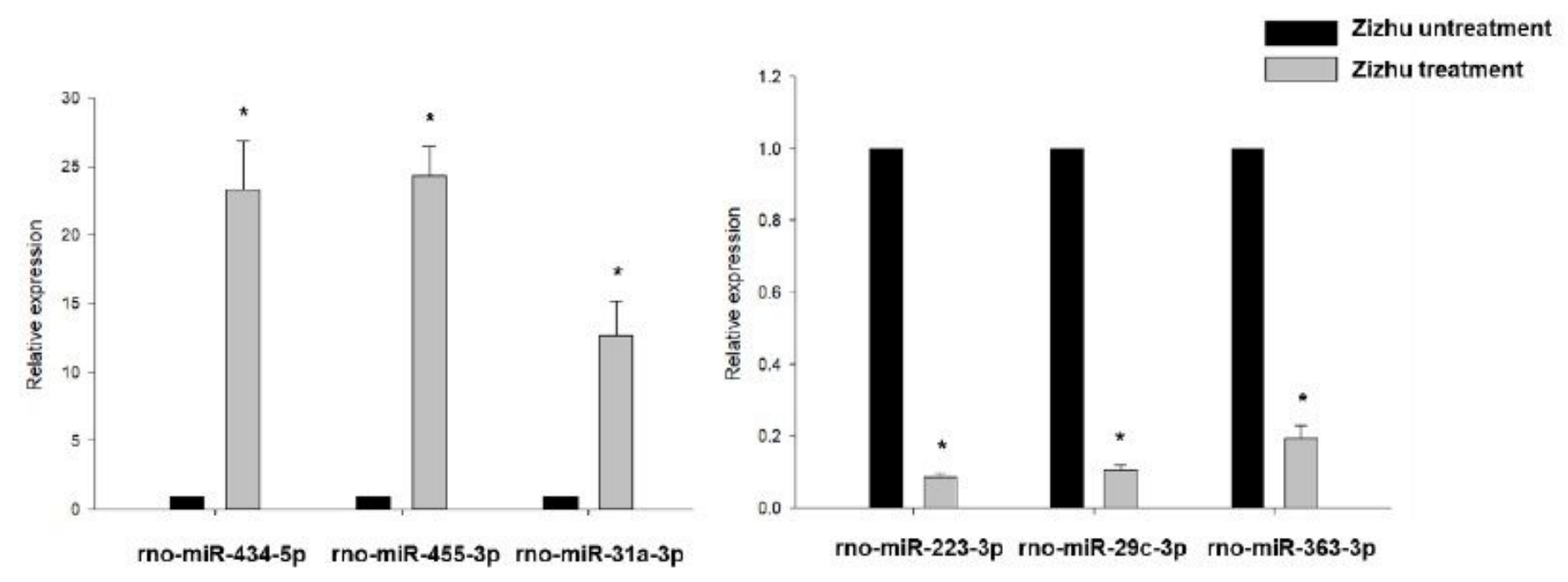

Figure 2

Validation of the identified differentially expressed miRNAs by qPCR. Asterisk represents $p$-value $<0.05$.

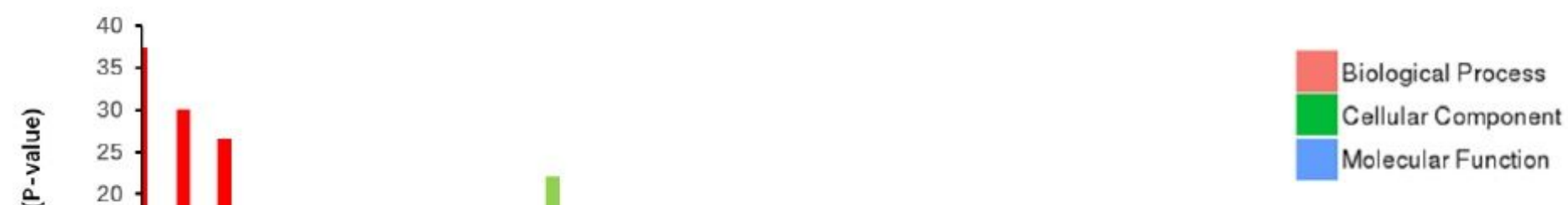

Figure 3

GO enrichment analysis of target genes. 


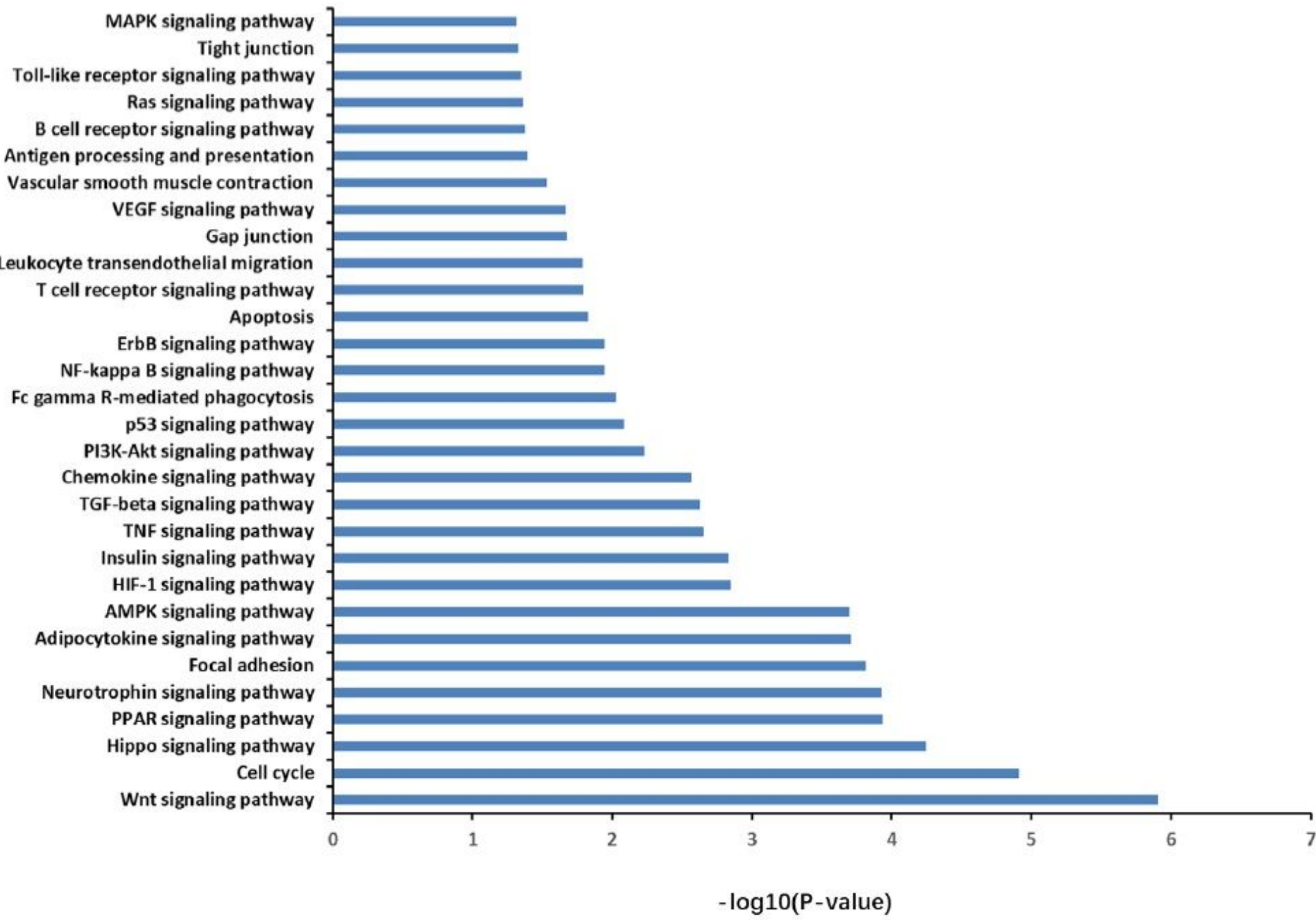

\section{Figure 4}

KEGG pathway enrichment analysis of target genes. 

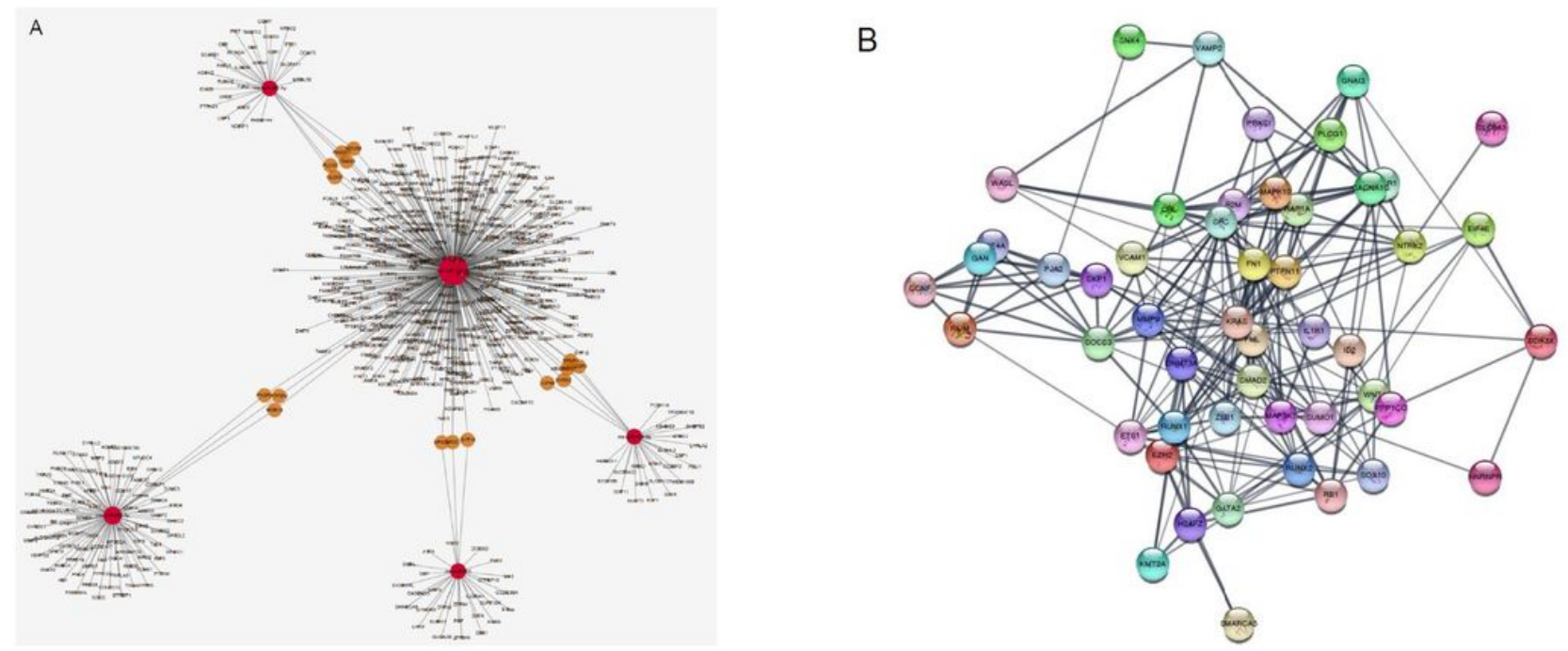

\section{Figure 5}

miRNA-target regulatory network. (A) Network of top 5 upregulated expression miRNAs with their targets. Red nodes represent upregulated miRNAs. Brown nodes represent their putative targets and links represent the regulation of miRNAs on their target genes. (B) The protein-protein interaction (PPI) network of target genes of the top 5 upregulated expression miRNAs with top 50 degree.
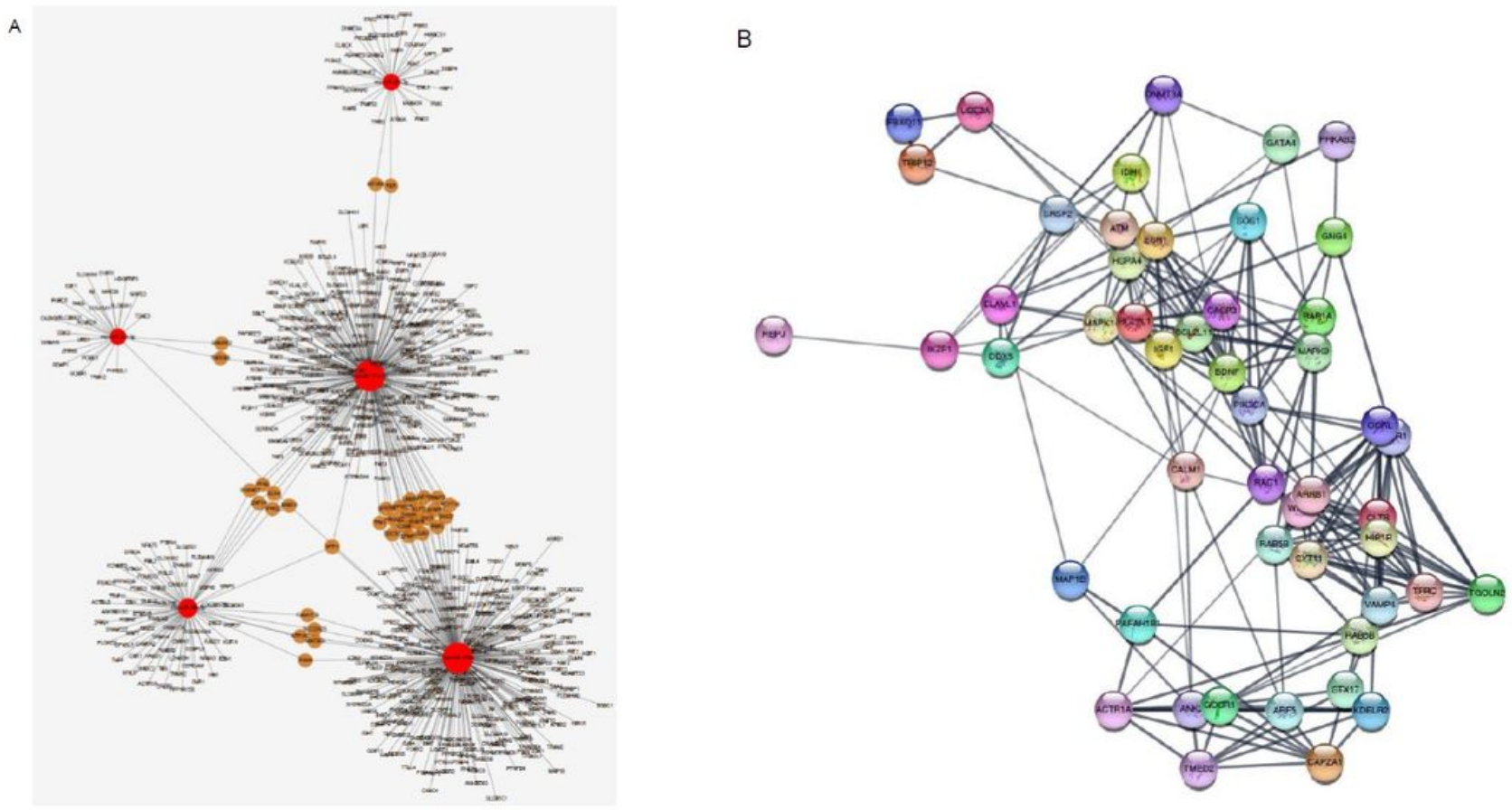


\section{Figure 6}

MiRNA-target regulatory network. (A) Network of top 5 downregulated expression miRNAs with their targets. Red nodes represent downregulated miRNAs. Brown nodes represent their putative targets and links represent the regulation of miRNAs on their target genes. (B) The protein-protein interaction (PPI) network of target genes of the top 5 downregulated expression miRNAs with top 50 degree. 